\title{
Adjusting the distance scales of old and intermediate-age tracers via statistical parallaxes
}

\author{
A. K. Dambis \\ Sternberg Astronomical Institute, M. V. Lomonosov Moscow State University, \\ Universitetskii pr. 13, Moscow, 119992 Russia \\ email: mirage@sai.msu.ru
}

\begin{abstract}
The method of statistical-parallax determination is used to adjust the photometric distance-scale zero points and test the selection of several samples of old and intermediate-age tracer objects in the Milky Way, including RR Lyrae- and Mira-type variables, blue and red horizontal-branch stars, and red giants. We obtain reasonable and stable absolute-magnitude (and, hence, photometric distance-scale) corrections for all samples except for the red horizontalbranch stars, which appear to be significantly contaminated by main-sequence turn-off and subgiant stars.
\end{abstract}

Keywords. stars: distances, stars: luminosities, stars: kinematics, stars: classification, stars: horizontal-branch, stars: variables: other

\section{Introduction}

We use the method of statistical-parallax determination in its rigorous maximumlikelihood version suggested by Murray (1983, p. 297) to analyze five tracer samples consisting (or supposedly consisting) of RR Lyrae variables, Mira variables, red-giant stars, and blue and red horizontal-branch (BHB, RHB) stars.

\section{RR Lyrae-type variables}

We use a bimodal version of the statistical-parallax method (for details, see Dambis 2009) to set the zero point of the metallicity- and period-luminosity (PL) relations, respectively, for Galactic field RR Lyrae-type variables. Our sample is composed of 368 stars with heliocentric distances $\leqslant 6.5 \mathrm{kpc}$, bona fide proper motions, radial velocities, and phase-corrected 2MAss $K$-band magnitudes (Cutri et al. 2003), as well as UCAC4 proper motions (Zacharias et al. 2012) and 355 stars with average $V$-band magnitudes. The resulting relations are

$$
\left\langle M_{V}\right\rangle=+1.17( \pm 0.09)+0.214[\mathrm{Fe} / \mathrm{H}]
$$

and

$$
\left\langle M_{K}\right\rangle=-0.80( \pm 0.08)-2.338 P_{\mathrm{F}}
$$

where the slopes have been adopted from Gratton et al. (2004) and Frolov \& Samus' (1998), respectively. Here, $P_{\mathrm{F}}$ is the period of fundamental-mode pulsation.

\section{Mira-type variables}

Our Mira sample is based on the catalog of Galactic Mira-type variables of Kharchenko et al. (2002), supplemented with radial-velocity data from Feast \& Whitelock (2000) and 


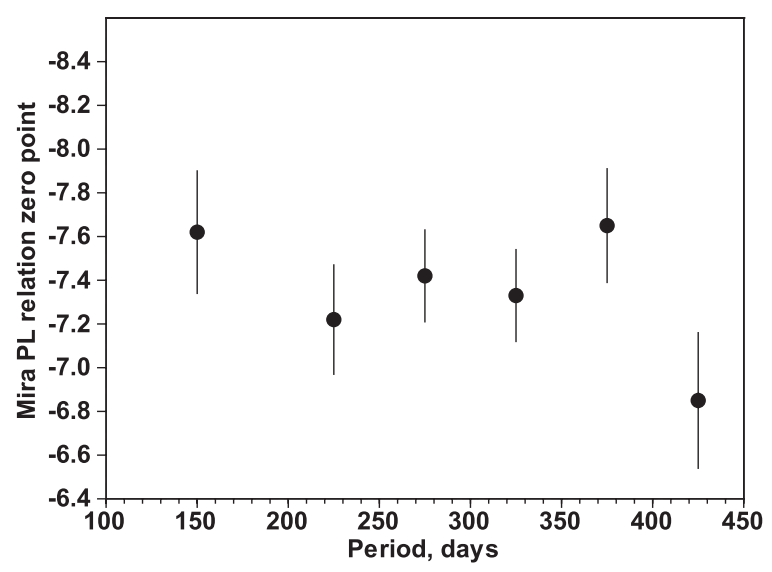

Figure 1. Zero point of the PL relation for Galactic Miras as a function of period.

Menzies et al. (2006), average $K$-band magnitudes adopted from Menzies et al. (2006), Whitelock et al. (2006, 2008), and 2MAss (Cutri et al. 2003), and proper motions from the UCAC4. The provisional distances to our sample Miras are based on the $\log P-M_{K}$ relation of Whitelock et al. (2008), $\left\langle M_{K}\right\rangle=-7.15-3.51(\log P-2.38)$. Using our inferred zero point, the relation has the following form:

$$
\left\langle M_{K}\right\rangle=-7.41( \pm 0.10)-3.51(\log P-2.38) \text {. }
$$

The absence of any apparent trend in the zero-point correction in the $\sim 120-400$ day period interval (see Fig. 1) implies that the slope of the PL relation for Galactic field Miras does not differ significantly from the corresponding slope for Miras in the Large Magellanic Cloud.

\section{Red giants}

Our third sample consists of 2519 red giants identified using SDSS/SEGUE data (Martell et al. 2011) with photometric distances based on absolute-magnitude calibration derived using 12 Gyr-old Dartmouth isochrones (Dotter et al. 2008). Fig. 2 shows the inferred zero-point correction of the adopted absolute-magnitude calibration as a function of metallicity. The adopted calibration and selection procedure appear robust given the moderate absolute-magnitude correction $\left(\Delta M_{g}=+0.17 \pm 0.06 \mathrm{mag}\right)$ and the lack of any apparent metallicity trend.

\section{Blue horizontal-branch stars}

Our fourth sample consists of 1955 rigorously selected blue horizontal-branch halo stars from sDSs data release (DR) 6 (Adelman-McCarthy et al. 2008) with photometric distances based on the calibration of Sirko et al. (2004) and radial velocities determined within the framework of the SEGUE project (Yanny et al. 2009). We adopted this sample from Xue et al. (2008) and supplemented it with absolute proper motions from the SDSS DR7 (Abazajian et al. 2009) database. We applied a single-mode version of the statisticalparallax method, parametrizing the variation of the velocity-ellipsoid parameters as a function of Galactocentric distance in terms of the model of Sommer-Larsen et al. (1997) and found the absolute-magnitude correction to be small $\left(\Delta M_{g}=-0.12 \pm 0.06 \mathrm{mag}\right)$ and highly stable across the entire metallicity range of BHB stars considered (see Fig. 3). 


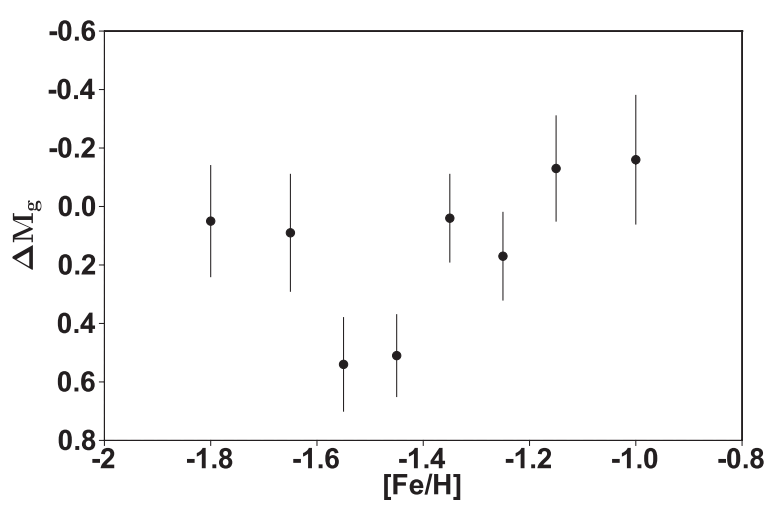

Figure 2. Zero-point correction of the absolute-magnitude calibration for red-giant stars based on the 12 Gyr-old Dartmouth isochrones as a function of metallicity.

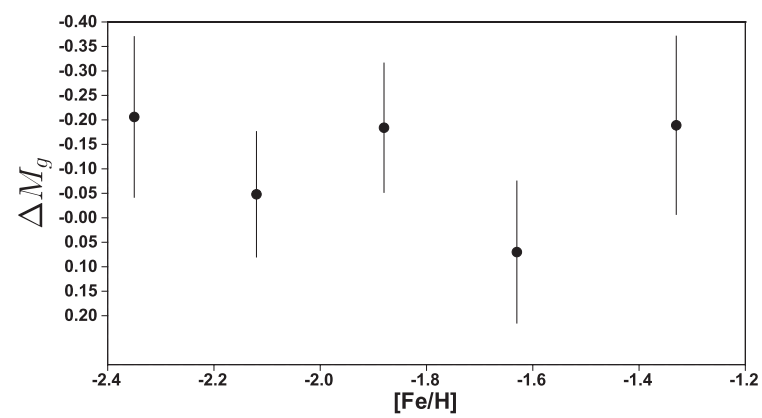

Figure 3. Zero-point correction to the absolute-magnitude calibration for BHB stars as a function of metallicity.

\section{Red horizontal-branch stars}

Our fifth sample consists of presumed field red horizontal-branch stars (5391 objects) selected by Chen et al. (2010) based on SDSS photometry and SEGUE spectroscopy using positions of stars in the metallicity-color and $T_{\text {eff }}-\log g$ diagrams. The surprising result is the average factor-of-two reduction in the adopted distances, implying a factor-of-four reduction in the adopted luminosities of the sample stars, rendering the horizontal-branch nature of most of the sample and the underlying selection procedure doubtful. To further investigate this issue, we determined the average absolute magnitudes, $M_{V}$, of stars in this sample in $(B-V)_{0}$ color bins and show the resulting color-magnitude diagram in Fig. 4, where the $\left[(B-V)_{0}, M_{V}\right]$ data points corresponding to our subsamples are superimposed on the 12 Gyr-old Dartmouth isochrone (Dotter et al. 2008). Our results appear to imply that the sample should consist mostly of main-sequence turn-off and subgiant stars and is perhaps only slightly contaminated by RHB stars, except for the metal-richest part.

\section{Acknowledgements}

This work was supported by the Russian Foundation for Basic Research (grants 1002-00489-a and 11-02-00608-a). 


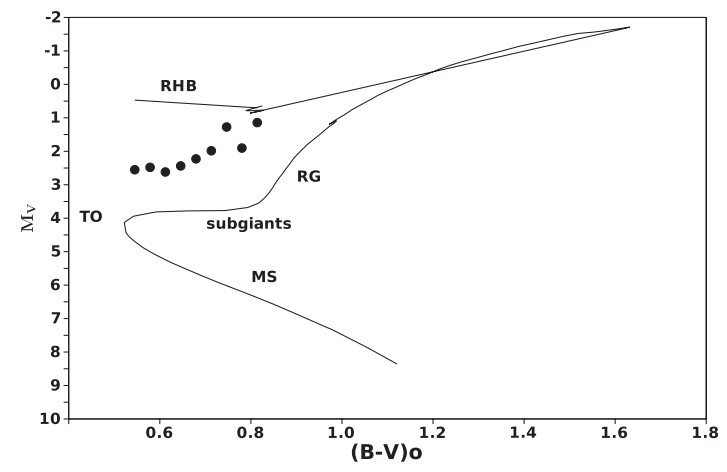

Figure 4. Inferred average positions of color-binned RHB subsamples (dots) on the $(B-V)_{0}$ versus $M_{V}$ color-absolute-magnitude diagram. Also indicated are the loci of main-sequence (MS), main-sequence turn-off (TO), red-giant (RG), and RHB stars, and the $Z=0.004,12$ Gyr-old Dartmouth isochrone.

\section{References}

Adelman-McCarthy, J. K., et al. 2008, ApJS, 175, 297

Abazajian, K. N., et al. 2009, ApJS, 182, 543

Chen, Y. Q., Zhao, G., Zhao, J. K., Xue, X. X., \& Schuster, W. J. 2010, AJ, 140, 500

Cutri, R. M., Skrutskie, M. F., van Dyk, S., et al. 2003, 2MASS All-Sky Catalog of Point Sources (CDS catalog II/246)

Dambis, A. K. 2009, MNRAS, 396, 553

Dotter, A., Chaboyer, B., Jevremovic, D., Kostov, V., Baron, E., \& Ferguson, J. W. 2008, ApJS, 178,89

Feast, M. W. \& Whitelock, P. A. 2000, MNRAS, 317, 460

Frolov, M. S. \& Samus', N. N. 1998, Astron. Lett., 24, 171

Gratton, R. G., Bragaglia, A., Clementini, G., Carretta, E., Di Fabrizio, L., Maio, M., \& Taribello, E. 2004, A\&\&A, 421, 937

Jones, R. V., Carney, B. W., Storm, J., \& Latham, D. 1992, ApJ, 385, 646

Kharchenko, N., Kilpio, E., Malkov, O., \& Schilbach, E. 2002, A\& $A, 384,925$

Martell, S. L., Smolinski, J. P., Beers, T. C., \& Grebel, E. K. 2011, A\&̊A, 534, 136

Menzies, J. W., Feast, M. W., \& Whitelock, P. A. 2006, MNRAS, 369, 783

Murray, C. A. 1983, Vectorial Astrometry (Bristol: A. Hilger)

Sirko E., Goodman J., Knapp G. R., et al. 2004, AJ, 127, 899

Sommer-Larsen J., Beers T. C., Flynn C., Wilhelm R., Christensen P. R. 1997, ApJ, 481, 755

Whitelock, P. A., Feast, M. W., Marang, F., \& Groenewegen, M. A. T.. 2006, MNRAS, 369, 751

Whitelock, P. A., Feast, M. W., \& van Leeuwen, F. 2008, MNRAS, 386, 313

Xue X. X., Rix H. W., Zhao G., et al. 2008, AJ, 684, 1143

Yanny, B., et al. 2009, AJ, 137, 4377

Zacharias, N., Finch, C. T., Girard, T. M., Henden, A., Bartlett, J. L., Monet, D. G., \& Zacharias, M. I. 2012, The fourth U.S. Naval Observatory CCD Astrograph Catalog (UCAC4) (CDS catalog I/322 ) 